\title{
Multimodality Imaging of Angiogenesis
}

\author{
Ho-Young Lee ${ }^{1}$ and Jae-Min Jeong ${ }^{*}, 2$ \\ ${ }^{I}$ Department of Nuclear Medicine, Seoul Metropolitan Boramae Center, Seoul, Republic of Korea \\ ${ }^{2}$ Department of Nuclear Medicine, College of Medicine, Seoul National University, Seoul, Republic of Korea
}

\begin{abstract}
Angiogenesis is an important biological process that is also related to various diseases, such as cancer, cardiovascular and cerebrovascular disease. Currently, the need for angiogenesis imaging is increasing due to the use of anti-angiogenic therapy to treat tumors, and of angiogenesis-inducing therapy to treat vascular diseases.

Several techniques can be used to visualize angiogenesis related parameters at the structural, functional, and molecular level, although clinically, most techniques involve structural or functional imaging. However, structural and functional parameters do not completely represent angiogenic activity, because they evaluate angiogenesis indirectly by measuring structural and functional changes. Molecular imaging techniques can be used to evaluate bio-markers directly related to angiogenesis, but have only recently been applied in clinical practice. It is now evident that assessment of angiogenesis at several different levels provides valuable information which could be used to individualize therapy and improve diagnosis.
\end{abstract}

Keywords: Angiogenesis, integrin, vascular endothelial growth factor.

\section{INTRODUCTION}

The term 'angiogenesis' was first used in 1787 by the British surgeon Dr. John Hunter, to describe blood vessel growth in the reindeer antler. Angiogenesis is an essential physiologic process, and is required for growth, development, and for wound healing. On the other hand, it is also a fundamental process that is required during the transition of tumors from a dormant to a malignant state. In 1968, an angiogenic diffusible factor was identified in tumors by Geenblatt and Shubik [1], and more recently, some biomarkers, such as integrin and VEGF were found to play key roles in the angiogenic process [2-4].

Today, angiogenesis is an important therapeutic target in cardiovascular and malignant diseases for different reasons. In cardiovascular or cerebrovascular diseases, the therapeutic goal is to induce angiogenesis to improve infarct tissue perfusion, and thus, to prevent reinfarction and to promote recovery from ischemic injury $[5,6]$. On the other hand, in the oncologic field angiogenic therapeutic strategies are based on the inhibition of angiogenesis, because tumor growth and metastasis are dependent on angiogenesis [7,8]. The dependency of tumor growth and metastasis on angiogenesis was first proposed by Dr. Judah Folkman in the New England Journal of Medicine in 1971, and initially this was regarded as heresy by leading physicians and scientists [9]. However, he is now regarded as a pathfinder of the use of anti-angiogenesis therapy in the oncology field. Based on his theory, many efforts have been made to develop antiangiogenic drugs. Initial efforts to develop anti-angiogenic drugs resulted in bevacizumab, which was later approved by

\footnotetext{
*Address correspondence to this author at the Department of Nuclear Medicine, College of Medicine, Seoul National University, 28 Yongondong, Chongno-gu, Seoul 110-744, Republic of Korea; Tel: +82-2-2072-
} 3805; Fax: +82-2-760-7690; E-mail: jmjng@snu.ac.kr the US Federal Drug Administration for the treatment of metastatic colorectal carcinoma. Subsequently, many drugs have undergone clinical trials and some have been approved for clinical usage. Furthermore, the increasing use of antiangiogenic drugs in the oncologic field has increased the importance of imaging the angiogenic process in terms of selecting those patients likely to respond to anti-angiogenic drugs.

In this review, we summarize the recent progress made in the imaging of angiogenesis and discuss their therapeutic and diagnostic applications in the oncologic and cardiovascular fields.

\section{STRUCTURAL IMAGING OF ANGIOGENESIS}

Angiogenesis results in the formation of a microvasculature. To evaluate angiogenesis, one could evaluate changes in the vascular volumes or densities of vessels. Currently, several experimental and conventional clinical imaging modalities, such as, computed (CT) angiography, contrast-enhanced ultrasound, high-resolution magnetic resolution angiography, and intravital microscopy can be used to acquire structural information about the microvasculature, although multidetector CT (MDCT) angiography is most useful for evaluating vascular structure.

MDCT angiography has better spatial and time resolutions than conventional CT, and enables larger scan volumes to be obtained in less time with better image quality. Because of its higher resolution, MDCT can be used to evaluate different vascular phases after contrast bolus administration [10], and it requires less contrast than conventional CT angiography. Accordingly, MDCT is the best available clinical modality for the evaluation of vascular structures surrounding nonvascular regions. However, because the size of a human capillary is about 7-10 $\mu \mathrm{m}$ and 
the spatial resolution of MDCT is about $1 \mathrm{~mm}$, MDCT is still inadequate at visualizing microvasculature structures [11].

The application of synchrotron light increases the number of photons produced per unit-area per second by bench top $x$-ray sources, and this improves spatial and contrast resolution. Furthermore, by using a combination of pure absorption and edge enhancement, Micro-CT makes it possible to visualize several cubic millimeters of microvasculature [12] at a resolution of several $\mu \mathrm{m}$ (Fig. 1) [13]. Accordingly, Micro-CT offers a valuable means of visualizing and evaluating the microvascular architecture, and of quantifying the vascular volumes and densities of vessels. However, this resolution image is obtained at the expense of long scan times and high x-ray doses, and thus, micro-CT is not suitable for repeated noninvasive vessel measurements. Flat panel volumetric CT (fPVCT) might be capable of overcoming these limitations of micro-CT [14], as it permits the acquisition of large volume slices per rotation, and has intrinsically higher resolution than MDCT. Kiessling et al. described a prototype fpVCT that combines the advantages of micro-CT and clinical CT scanners and used it to acquire high-resolution images at the experimental and preclinical in vivo levels [15]. The spatial resolution of fpVCT is about $45 \mu \mathrm{m}$ with isotropic voxels of less than 4 . $10^{-4} \mathrm{~mm}^{3}$, and the further development of fpVCT could result in systems with the advantages of clinical MDCT and preclinical micro-CT.

Furthermore, micro-CT systems could be used to study the effect of anti-angiogenic drugs at the microvascular level [16], but it can only be used to evaluate the results of angiogenesis, and not the angiogenic process.

\section{FUNCTIONAL IMAGING OF ANGIOGENESIS}

The functional imaging of angiogenesis is a tool to evaluate physiologic parameters, such as, perfusion and blood flow, which are related to hemodynamic changes. Dynamic contrastenhanced MRI could be used to evaluate the microvasculature noninvasively by allowing the tracking of the pharmacokinetics of an injected contrast agent passing through a tumor [17].
Because it allows contrast agent passage to be evaluated, dynamic contrast-enhanced MRI could be used to measure changes in hemodynamic parameters, such as, diffusion, perfusion, extravascular and vascular volumes, and blood flow. Dynamic contrast-enhanced MRI has also been applied to measure changes in hemodynamic parameters during an evaluation of the effect of anti-angiogenic drugs [18]. However, the hemodynamic changes observed were not found to be significantly correlated with clinical outcome, especially in terms of response assessment, though clinical outcomes appeared to depend strongly on the therapeutic protocol used and tumor type $[19,20]$. One reason for this discordance between hemodynamic changes and clinical outcome might be that MRI measurements only indirectly represent changes in angiogenesis.

Ultrasound is widely used in clinical practice to evaluate blood flow through organs or tumors. Due to its simplicity, ease of use, speed, and safety, ultrasound imaging is being increasingly used to monitor angiogenesis for diagnosis, treatment assessment, follow-up, and therapy guidance. Technically, Doppler and microbubble ultrasound can be used to evaluate microcirculations, whereas power Doppler can be used to estimate relative fractional vascular volumes and blood velocities. Currently, ultrasound imaging is able to measure blood flow in the microvasculature in vessels of less than $100 u \mathrm{~m}$. High frequency pulsed Doppler ultrasound allows blood flow in arterioles as small as $15 u \mathrm{~m}$ to be directly assessed [21]. However, power Doppler has low reproducibility and shows high individual variations, and is unsuitable for the evaluation of many lesions. Ultrasound using microbubbles can measure the blood flow in the microcirculation level by increasing the signal from smaller vessels. However, microbubbles have diameters of 1-10 um, which are larger than the particle sizes of contrast agents. Accordingly, the use of ultrasound allows one to access the properties of microvascular compartments related to larger dimensions, but it lacks the spatial resolution to allow evaluations of microvasculature morphologies and flow dynamics.

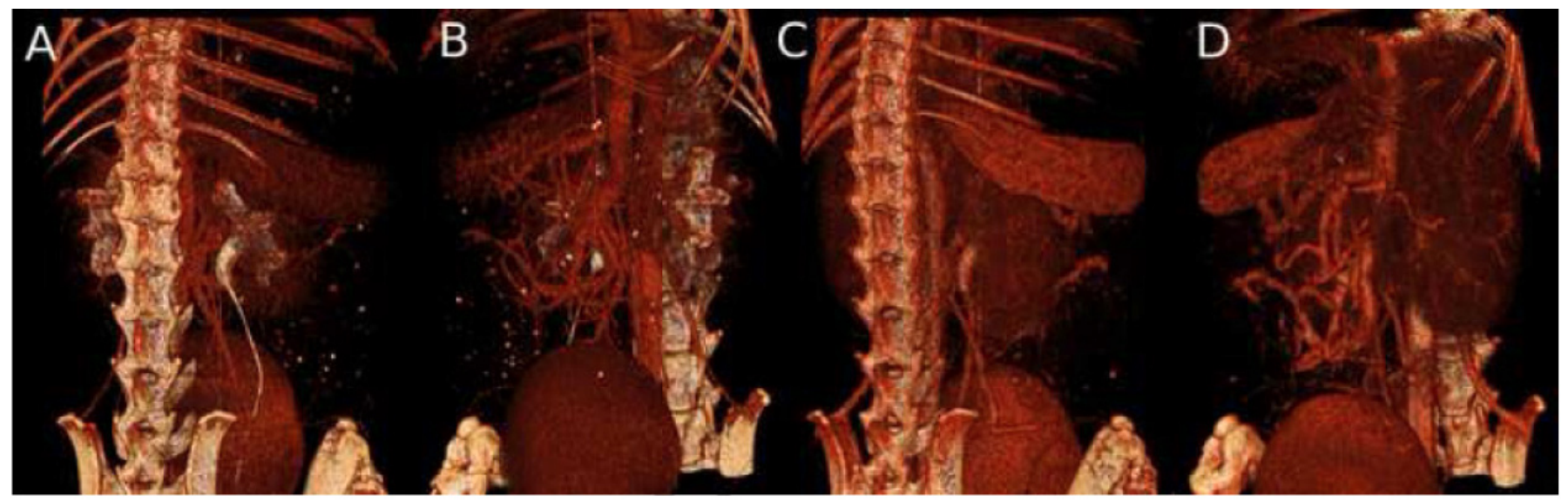

Fig. (1). Volume rendering of murine abdominal vasculature after the administration of a first (A+B) and second (B+C) bolus of $500 \mu 1$ Fenestra $\mathrm{VC}^{\circledR}$. Jejunal, ileal, ileocolic and mesenteric veins feeding the portal vein can clearly be visualized in both datasets, with higher vessel contrast in $\mathbf{C}+\mathbf{D}$. Corresponding arteries running alongside the veins are only visible up to the level of mesenteric arteries in both ungated datasets due to motion blurring. The early enhancements of the kidneys and ureter were due to the injection of a few microliters of a conventional contrast agent prior to the injection of a more expensive blood pool contrast agent to ensure correct catheter placement (reprinted with permission of [13]). 
Optical imaging is one of the most powerful in vivo molecular imaging modalities. It is a highly sensitive method that offers single-cell resolution and real-time imaging, and thus, allows the biological interactions that are critical to tumor development and angiogenesis to be monitored. Optical imaging can be used to evaluate vascular permeability, vessel size, and blood flow [22, 23], and in combination with fluorescent dyes and quantum dots to produce clear images of blood vessels. Several highresolution microscopic optical imaging techniques, such as, intravital microscopy, confocal laser scanning microscopy, multiphoton laser scanning microscopy, and in situ scanning force microscopy have been developed to study molecular events in vivo [24, 25]. Despite the high spatial resolution and clarity of optical imaging, it is intrinsically limited by a lack of depth penetration due to light scattering and absorption by tissues.

Single photon emission computed tomography (SPECT) is a useful modality for evaluating perfusion and blood flow in normal organs and in tumor models, and has already been used in clinical practice to evaluate brain, heart, and tumor perfusion. Furthermore, the uptake of SPECT radiotracers is linearly correlated with perfusion in organs and tumors. Myocardial and brain SPECT have already been used to evaluate the effects of angiogenesis in infarct areas [26]. However, SPECT has a relatively low spatial resolution and involves the use of a radioisotope.

\section{MOLECULAR IMAGING OF ANGIOGENESIS}

Due to advances in imaging techniques, such as, dynamic contrast-enhanced MRI, it could be used to assess many hemodynamic parameters and microvascular structures. However, the results of studies performed so far have not revealed the biological mechanism underlying angiogenesis. Consequently, more specific imaging techniques are required that represent angiogenic activity, and the need for such imaging techniques is increasing due to the increasing usage of drugs that target angiogenesis. Evaluations of angiogenic activities are necessary for pre-therapeutic anti-angiogenesis assessments and for post-therapy response evaluations. The molecules known to regulate angiogenesis include; growth factor receptors, tyrosine kinase receptors, G-proteincoupled receptors for angiogenesis modulating proteins, integrins, and matrix metalloproteinases; and these molecules could be utilized as targets for specific angiogenesis imaging. Of these target molecules, integrin $\alpha_{v} \beta_{3}$ and VEGF receptors (VEGFRs) are most widely used for angiogenesis-imaging studies.

Integrins are receptors that mediate cell adhesion between cells and adjacent tissues, which could be other cells or extracellular membranes. On the other hand, integrins play important roles during angiogenesis and metastasis $[27,28]$, for example, during tumor angiogenesis, integrins are expressed on endothelial cells and regulate cell migration, metastasis, and survival. Furthermore, integrins on tumor cells induce metastasis by facilitating cell migration across blood vessels, and integrin $\alpha_{v} \beta_{3}$ is known to be significantly overexpressed on new vessels around tumors but not on normal endothelial cells $[29,30]$. Integrin $\alpha_{\mathrm{v}} \beta_{3}$ binds to arginine-glycineaspartic acid (RGD) a component of the extracellular matrix, and by using appropriate cyclic
RGD peptides and monoclonal antibodies against integrin $\alpha_{\mathrm{v}} \beta_{3}$, multimodality imaging techniques, such as, PET, SPECT, MRI, ultrasound, and optical techniques could be used to evaluate angiogenesis by quantifying integrin $\alpha_{v} \beta_{3}$ expression (Fig. 2) [30-34]. In particular, ${ }^{18} \mathrm{~F}$-galacto-RGD and ${ }^{68} \mathrm{Ga}$-RGD PET have been evaluated in patients, and showed good contrast at lower radiation doses than ${ }^{18} \mathrm{~F}-\mathrm{FDG}$ [34-37]. Furthermore, RGD uptake was found to be significantly correlated with $\alpha_{v} \beta_{3}$ expression in a mouse tumor model and in cancer patients.

When ${ }^{68} \mathrm{Ga}$-RGD was used the fast blood clearance of most monomeric RGD peptide-based tracers resulted in relatively low tumor uptake and rapid tumor washout, which might have been due to suboptimum receptor-binding affinity/selectivity and inadequate contact with integrin $\alpha_{v} \beta_{3}$ binding sites in the extracellular matrix. Multimerization of cyclic RGD peptides can be used to delay the clearance time of RGD, and the multimerization of cyclic RGD has been reported to improve affinity for integrin $\alpha_{v} \beta_{3}$, and thus, to significantly improve tumor targeting as compared with monomeric RGD analogs $[38,39]$. Furthermore, the multimerization of cyclic RGD peptides was found to visualize low to medium integrin expression tumors [38]. Several dimeric and multimeric RGD peptide-based imaging probes are in the process of clinical translation for first human studies. Integrin $\alpha_{v} \beta_{3}$ is expressed not only on neovasculature, but also in tumor cells, and therefore, small molecules, such as, RGD antibody targeting integrin $\alpha_{v} \beta_{3}$, do not provide accurate information regarding tumor angiogenesis because such probes bind to integrin $\alpha_{v} \beta_{3}$ expressed on the tumor vasculature and on tumor cells. To visualize expression on neovasculature alone, integrintargeted nanoparticles are used for angiogenesis imaging, and because of their sizes and rigidities, integrin-targeted nanoparticles do not extravasate, and therefore, may be close to ideal vascular integrin-specific probes.

The VEGF/VEGFR signaling pathway plays a pivotal role during the development of the normal vasculature and during many disease processes. The VEGF family is composed of 7 members with a common VEGF homology domain. The angiogenic actions of VEGF are mediated mainly through VEGFR-1 and VEGFR-2, which are both largely restricted to vascular endothelial cells. Furthermore, all VEGF-A isoforms are ligands of VEGFR-1 and VEGFR2 , the latter of which mainly mediates the angiogenic, mitogenic, and permeability-enhancing effects of VEGF. Due to the key role of VEGF-A during cancer progression, it has been targeted for cancer treatment, and humanized antiVEGF monoclonal antibody bevacizumab (Avastin; Genentech) has been approved by the FDA as a first-line treatment [40]. Many radionuclide and non-radionuclide based VEGFR imaging investigations were conducted after the successful initial clinical evaluation of ${ }^{123}$ I-VEGF165 in patients with gastrointestinal cancer [41, 42]. Furthermore, the developments of VEGF and VEGFR-targeted molecular imaging probes would provide a new means of assessing anti-angiogenic therapies and of clarifying the role of VEGF/VEGFR in angiogenesis-related diseases. Imaging probes based on wild-type VEGF-A isoforms bind to both VEGFR-1 and -2, and furthermore, VEGFR-1 expression is high in kidneys that take up VEGF-A based tracers. However, VEGFR-2 is more functionally important during 

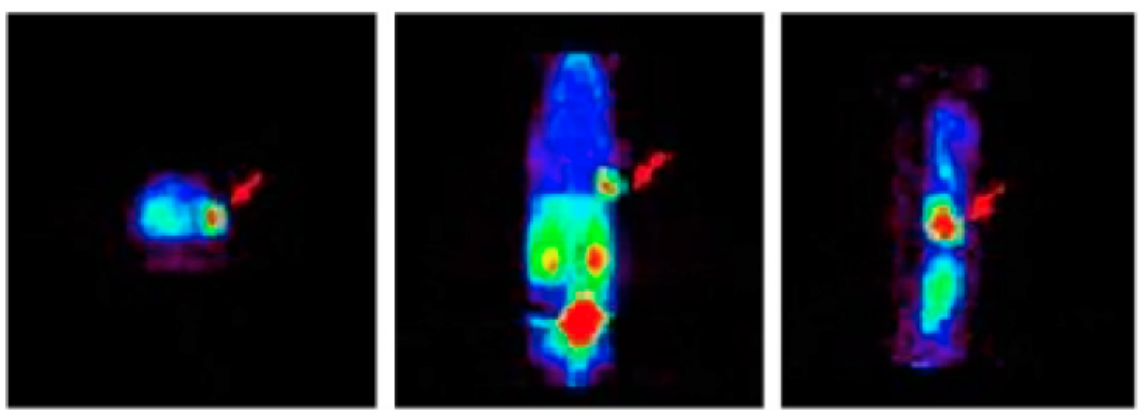

\section{$1 \mathrm{~h}$}
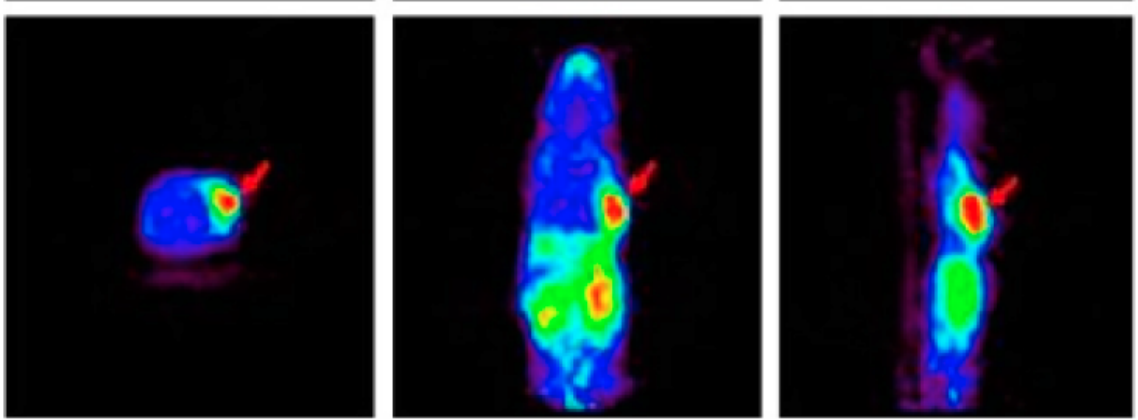

\section{$2 \mathrm{~h}$}

Fig. (2). Small-animal PET of ${ }^{68} \mathrm{Ga}-\mathrm{NOTA}-\mathrm{RGD}$ injected mice bearing SNU-C4 xenografts at 1 and $2 \mathrm{~h}$ after injection without cold $\mathrm{c}(\mathrm{RGDyK})(\mathbf{A})$ or with cold c(RGDyK) $(60 \mathrm{mg})(\mathbf{B})$. Images at $1 \mathrm{~h}$ were taken before micturition, and images at $2 \mathrm{~h}$ after micturition. Arrows indicate tumor positions. The acquisition time used was $20 \mathrm{~min}$ (reprinted with permission of [44]).

cancer progression, and the visualization of VEGFR-2 is valuable for evaluating patients with malignancies prior to the commencement of anti-VEGFR-2 therapy. Many efforts have been made to develop VEFGR-2 specific probes, based on screening experiments and the structures and affinities of mutant VEGF-A or antiVEGFR-2 antibody [43, 44]. Further improvements in VEGFR-2 binding affinity, specificity, pharmacokinetics, and tumor-targeting efficacy are necessary before VEGF-based imaging probes can be utilized in clinical practice.

\section{CONCLUSION}

Currently, many imaging techniques can be used to visualize various aspects of angiogenesis. Furthermore, the need for angiogenesis imaging techniques is increasing due to the increasing use of anti-angiogenic drugs and the developments of new drugs. Recently, functional hemodynamic vascular parameters, such as, blood flow and blood volume, have been used clinically to assess treatment effects on angiogenesis. However, the functional hemodynamic parameters examined only indirectly represent angiogenesis. On the other hand, molecular imaging offers the possibility of evaluating angiogenic activity at the molecular level. However, there remains a need for more specific target structures for the assessment of angiogenesis. Nevertheless, assessments of different parameters of angiogenesis at the structural, functional, and molecular levels for clinical purposes will undoubtedly result in further anti-angiogenic therapy improvements.

\section{REFERENCES}

[1] Greenblatt M, Shubi P. Tumor angiogenesis: transfilter diffusion studies in the hamster by the transparent chamber technique. J Nat Cancer Inst 1968; 41: 111-24.

[2] Silva R, D'Amico G, Hodivala-Dilke KM, Reynolds LE. Integrins: the keys to unlocking angiogenesis. Arterioscler Thromb Vasc Biol 2008; 28: 1703-13.
[3] Thurston G, Kitajewski J. VEGF and Delta-Notch: interacting signaling pathways in tumour angiogenesis. Br J Cancer 2008; 99: 1204-9.

[4] Hendriksen EM, Span PN, Schuuring J, et al. Angiogenesis, hypoxia and VEGF expression during tumour growth in a human xenograft tumour model. Microvasc Res 2009; 77: 96-103.

[5] Koketsu N, Berlove DJ, Msokowitz MA, Kowall NW, Caday CG, Finkelstein SP. Pretreatment with intraventricular basic fibroblast growth factor decreases infarct size following focal cerebral ischemia in rats. Ann Neurol 1994; 35: 451-7.

[6] Stegmann TJ. New approaches to coronary heart disease: induction of neovascularisation by growth factors. BioDrugs 1999; 11: 301-8.

[7] Brown JM, Giaccia AJ. The unique physiology of solid tumors: opportunities (and problems) for cancer therapy. Cancer Res 1998; 58: $1408-16$

[8] Bergers G, Benjamin LE. Tumorigenesis and the angiogenic switch. Nat Rev Cancer 2003; 3: 401-10.

[9] Folkman J. Tumor angiogenesis: therapeutic implications. N Engl J Med 1971; 285: 1182-6.

[10] Ippolito D, Sironi S, Pozzi M, et al. Hepatocellular carcinoma in cirrhotic liver disease: functional computed tomography with perfusion imaging in the assessment of tumour vascularization. Acad Radiol 2008; 15: 919-27.

[11] Engelke C, Schaefer-Prokop C, Schirg E, Freihorst J, Grubnic S, Prokop M. High-resolution CT and CT angiography of peripheral pulmonary vascular disorders. Radiographics 2002; 2: 739-64.

[12] Witeholt C, Roerig DL, Gordon JB, Haworth ST, Molthen RC, Clough AV. Bronchial circulation angiogenesis in the rat quantified with SPECT and micro-CT. Eur J Nucl Med Mol Imaging 2008; 35: 1124-32.

[13] Schambach SJ, Bag S, Groden C, Schillig L, Brockmann MA. Vascular imaging in small rodents using micro-CT. Methods 2010; 50: 26-35.

[14] Greschus S, Kiessling F, Lichy MP, et al. Potential applications of flat-panel volumetric $\mathrm{CT}$ in morphologic and functional small animal imaging. Neoplasia 2005; 7: 730-40.

[15] Kiessling F, Greschus S, Lichy MP, et al. Volumetric computed tomography (VCT): a new technology for noninvasive, high resolution monitoring of tumour angiogenesis. Nat Med 2004; 10: 1133-8.

[16] Shen X, Wan C, Ramaswamy G, et al. Prolyl hydroxylase inhibitors increase neoangiogenesis and callus formation following femur fracture in mice. J Orthop Res 2009; 27: 1298-305. 
[17] Turkbey B, Kobayashi H, Ogawa M, Bernardo M, Choyke PL. Imaging of tumor angiogenesis: functional or targeted? AJR Am J Roentgenol 2009; 193: 304-13.

[18] Bonekamp D, Macura KJ. Dynamic contrast-enhanced magnetic resonance imaging in the evaluation of the prostate. Top Magn Reson Imaging 2008; 19: 273-84.

[19] Jain RK, Duda DG, Willett CG, et al. Biomarkers of response and resistance to antiangiogenic therapy. Nat Rev Clin Oncol 2009; 6: $327-38$.

[20] Baar J, Silverman P, Lyons J, et al. A vasculature-targeting regimen of preoperative docetaxel with or without bevacizumab for locally advanced breast cancer: impact on angiogenic biomarkers. Clin Cancer Res 2009; 15: 2583-90.

[21] Ferrara KW, Merritt CR, Burns PN, Foster FS, Mattrey RF, Wichkline SA. Evaluation of tumour angiogenesis with US: imaging, Doppler, and contrast agents. Acad Radiol 2000; 7: 82439.

[22] Dedeugd C, Wankhede M, Sorg BS. Multimodal optical imaging of microvessel network convective oxygen transport dynamics. Appl Opt 2009; 48: D187-97.

[23] McDonald DM, Choyke PL. Imaging of angiogenesis: from microscope to clinic. Nat Med 2003; 9: 713-25.

[24] Brown EB, Campbell RB, Tsuzuki Y. In vivo measurement of gene expression, angiogenesis, and physiological function in tumors using multiphoton scanning microscopy. Nat Med 2001; 7: 864-8.

[25] Dunphy MP, Entenberg D, Toledo-Crow R, Larson SM. In vivo microcartography and subcellular imaging of tumour angiogenesis: a novel platform for translational angiogenesis research. Microvasc Res 2009; 78; 51-6.

[26] Cho KR, Choi JS, Hahn W, et al. Therapeutic angiogenesis using naked DNA expressing two isoforms of the hepatocyte growth factor in a porcine acute myocardial infarction model. Eur $\mathbf{J}$ Cardiothorac Surg 2008; 34: 857-63.

[27] Hynes RO. Integrins: bidirectional, allosteric signaling machines. Cell 2002; 110: 673-87.

[28] Hood JD, Cheresh DA. Role of integrins in cell invasion and migration. Nat Rev 2002; 2: 91-100.

[29] Hodivala-Dilke K. Alphavbeta3 integrin and angiogenesis: a moody integrin in a changing environment. Curr Opin Cell Biol 2008; 20: 514-19.

[30] Haubner R. Alphavbeta3-integrin imaging: a new approach to characterise angiogenesis? Eur J Nucl Med Mol Imaging 2006; 33(Suppl 1): 54-63.
[31] Cai W, Rao J, Gambhir SS, Chen X. How molecular imaging is speeding up antiangiogenic drug development. Mol Cancer Ther $2006 ; 5 ; 2624-33$.

[32] Cai W, Gambhir SS, Chen X. Multimodality tumor imaging targeting integrin avb3. Biotechniques 2005; 39 suppl: 6-17.

[33] Choe YS, Lee KH. Targeted in vivo imaging of angiogenesis: present status and perspectives. Curr Pharm Des 2007; 23: 17-31.

[34] Haubner R, Decristoforo C. Radiolabelled RGD peptides and peptidomimetics for tumour targeting. Front Biosci 2009; 14: 87286.

[35] Beer AJ, Haubner R, Goebel $\mathrm{M}$, et al. Biodistribution and pharmacokinetics of the alphavbeta3-selective tracer ${ }^{18} \mathrm{~F}$-galactoRGD in cancer patients. J Nucl Med 2005; 46: 1333-41.

[36] Haubner R, Kuhnast B, Mang C, et al. $\left[{ }^{18} \mathrm{~F}\right]$ Galacto-RGD: synthesis, radiolabeling, metabolic stability, and radiation dose estimates. Bioconjug Chem 2004; 15: 61-9.

[37] Jeong JM, Hong MK, Chang YS, et al. Preparation of a promising angiogenesis PET imaging agent: ${ }^{68} \mathrm{Ga}$-labeled c(RGDyK)isothiocyanatobenzyl-1,4,7-triazacyclononane-1,4,7-triacetic acid and feasibility studies in mice. J Nucl Med 2008; 49: 830-36.

[38] Li ZB, Cai W, Cao Q, et al. ${ }^{64} \mathrm{Cu}$-labeled tetrameric and octameric RGD peptides for small-animal PET of tumor alpha(v)beta(3) integrin expression. J Nucl Med 2007; 48: 1162-71.

[39] Cacciari B, Spalluto G. Non peptidic avb3 antagonists: recent developments. Curr Med Chem 2005; 12: 51-70.

[40] Ferrara N, Hillan KJ, Gerber HP, Novotny W. Discovery and development of bevacizumab, an anti-VEGF antibody for treating cancer. Nat Rev Drug Discov 2004; 3: 391-400.

[41] Li S, Peck-Radosavljevic M, Kienast $\mathrm{O}$, et al. Iodine-123 vascular endothelial growth fracotr-165 ( ${ }^{123} \mathrm{I}$-vegf 165), biodistribution, safety and radiation dosimetry in patients with pancreatic cancer. Q J Nucl Med Mol Imaging 2004; 48: 198-206.

[42] Li S, Peck-Radosavljevic M, Kienast $\mathrm{O}$, et al. Imaging gastrointestinal tumours using vascular endothelial growth fractor165 (VEGF 165) receptor scintigraphy. Ann Oncol 2003; 14: 12747.

[43] Keyt BA, Nguyen HV, Berleau LT, et al. Identification of vascular endothelial growth factor determinants for binding KDR and FLT-1 receptors: generation of receptor-selective VEGF variants by sitedirected mutagenesis. J Biol Chem 1996; 271: 5638-46.

[44] Wang H, Cai W, Chen K, et al. A new PET tracer specific for vascular endothelial growth factor receptor 2. Eur J Nucl Med Mol Imaging 2007; 34: 2001-10.

This is an open access article licensed under the terms of the Creative Commons Attribution Non-Commercial License (http://creativecommons.org/licenses/by-nc/ 3.0/) which permits unrestricted, non-commercial use, distribution and reproduction in any medium, provided the work is properly cited. 